\title{
Biochemical parameters response to weight loss in patients with non-alcoholic steatohepatitis.
}

\author{
Shehab M. Abd El-Kader ${ }^{1}$, Fadwa M. Al-Shreef², Osama H. Al-Jiffri ${ }^{2}$
}

1. Department of Physical Therapy, Faculty of Applied Medical Sciences, King AbdulazizUniversity.

2. Department of Medical Laboratory Technology, Faculty of Applied Medical Sciences, King Abdulaziz University.

\begin{abstract}
Background: Non-alcoholic steatohepatitis (NASH) is a chronic liver disease that is capable of progressing to end-stage liver disease, but generally has a benign course. Non-alcoholic steatohepatitis (NASH) is a growing public health problem with no approved therapy. NASH projected to be the leading cause of liver transplantation in the United States by 2020. Obesity, non-insulin-dependent diabetes mellitus and hyperlipidaemia are the most common associations of the disease. Global prevalence of NASH is $10-24 \%$ amongst general population but increases to $25-75 \%$ in obese diabetic individuals. Objective: There is an urgent need for efficient therapeutic options as there is still no approved medication. The aim of this study was to detect changes in biochemical parameters including insulin resistance, cytokines, blood lipid profile and liver enzymes following weight loss in patients with non-alcoholic steatohepatitis.

Materials and methods: One hundred obese patients with NASH, their age between 35-50 years, body mass index (BMI) from 30 to $35 \mathrm{Kg} / \mathrm{m}^{2}$ were included in the study in two subgroups; the first group (A) received moderate aerobic exercise training in addition to diet regimen, where the second group (B) received no treatment intervention.

Results: The mean values of leptin, TNF- $\alpha$, IL6, IL8, Alanine Aminotransferase (ALT), Aspartate Aminotransferase (AST), Homeostasis Model Assessment-Insulin Resistance- index (HOMA-IR), Total Cholesterol (TC), Low Density Lipoprotein Cholesterol (LDL-c), Triglycerides (TG) and BMI were significantly decreased in group (A), where the mean value of Adiponectin and High Density Lipoprotein Cholesterol (HDL-c) were significantly increased, while there were no significant changes in group (B). Also, there was a significant difference between both groups at the end of the study.

Conclusion: Weight loss modulates insulin resistance, adiponectin, leptin, inflammatory cytokine levels and markers of hepatic function in patients with nonalcoholic steatohepatitis.

Keywords: Weight loss, insulin resistance, cytokines, liver enzymes, nonalcoholic steatohepatitis.

DOI: http://dx.doi.org/10.4314/ahs.v16i1.32

Cite as: Abd El-Kader SM, Al-Shreef FM, Al-Jiffri OH. Biochemical parameters response to weight loss in patients with non-alcoholic steatohepatitis. Afri Health Sci. 2016;16(1): 242-249. bttp://dx.doi.org/10.4314/abs.v16i1.32
\end{abstract}

\section{Introduction}

Non-alcoholic steatohepatitis (NASH) is a pathological condition characterized by accumulation of lipids in the liver of non-alcoholic individuals and consequent oxidative stress leading to cirrhosis of liver in the long run ${ }^{1 .}$ Incidence of NASH in USA is 30\% of the adult population $^{2}$. Even, in Asian countries, number of individuals

\section{Corresponding author: \\ Shehab Mahmoud Abd El- Kader, Faculty of Applied Medical Sciences, Department of Physical Therapy, King Abdulaziz University, P.O. Box 80324, Jeddah, 21589, Saudi Arabia. Phone: +966-569849276 \\ Email: salmuzain@live.com}

being diagnosed with NASH is on the rise $\mathrm{e}^{3}$. The clinical importance of NASH is due to its potential to progress to liver cirrhosis, hepatocarcinoma and liver failure 4 . Non-alcoholic steatohepatitis is a growing public health problem with no approved therapy and projected to be the leading cause of liver transplantation in the United States by $2020^{5}$.

Obese individuals are at maximum risk of developing $\mathrm{NASH}^{6}$ and a strong positive correlation exists between insulin resistance (IR) and development of NASH as obesity, non-insulin-dependent diabetes mellitus and hyperlipidaemia are the most common associations of the disease. Persons with central obesity are at particular risk ${ }^{7}$. The prevalence of NASH is estimated to be as high as $40-100 \%$ in obese adults worldwide ${ }^{8}$. 
Non alcoholic steatohepatitis is characterized by diffuse fatty acid infiltration and inflammation. The exact cause of NASH is unclear, but it is increasingly becoming more evident that the disease is much more common than was previously thought ${ }^{4}$. Inflammatory cytokines, IL- 6 , TNF- $\alpha$ and IL- 8 , and oxidative stress markers were significantly higher in the non-alcoholic steatohepatitis patients ${ }^{9}$.

The pathogenesis of NASH includes insulin resistance, increased inflammation, tumor necrosis factor-alpha (TNF- $\alpha$ ), interleukin-6 (IL-6) and increased oxidative damage ${ }^{10-12}$. Moreover, leptin and adiponectin have been implicated in the pathogenesis of both non-alcoholic steatohepatitis and insulin resistance ${ }^{13}$. Tumor necrosis factor- $\alpha$ and other TNF-induced cytokines such as interleukin- 6 and interleukin- 8 levels were higher in animals with NASH. This is likely related to the progression of NASH in liver cirrhosis ${ }^{14}$.

In many cases, NASH causes asymptomatic elevation of plasma aspartate transaminase (AST) and alanine transaminase (ALT). However, ALT is most closely related to liver fat accumulation, and it has been used as an indicator of $\mathrm{NASH}^{15}$. Moreover, insulin resistance plays a major role in the development of $\mathrm{NASH}^{16}$. However, serum concentrations of tumor necrosis factor-alpha $(\mathrm{TNF}-\alpha)$ are related to the severity of insulin resistance and to central adiposity ${ }^{17}$.

The aim of this study was to detect changes in biochemical parameters including TNF- $\alpha$, IL- 6 , IL- 8 , Leptin, Adiponectin, Insulin resistance, ALT, AST, TC, LDL-c, TG and HDL-c following weight loss in patients with nonalcoholic steatohepatitis.

\section{Subjects, materials and methods Subjects}

One hundred obese nonalcoholic steatohepatitis patients of both sexes ( 50 males \& 50 females) were selected from the Internal Medicine Department at King Abdul Aziz University Hospital and other Hospitals at Jeddah area. Their age was between $45-58$ years, the body mass index (BMI) ranged from 30 to $35 \mathrm{Kg} / \mathrm{m}^{2}$. Subjects with a history of smoking, cardiovascular disease, alcohol abuse problems, hepatic (i.e. infectious and viral disease) or renal disease were excluded from the study. Each patient underwent abdominal US (Siemens Antares equipment with $\mathrm{CH} 4-1 \mathrm{MHz}$ transducer; Siemens Medical, Erlangen, Germany) in a fasting state.
The presence of hepatic steatosis was assessed independently by radiologists according to findings such as hepatorenal contrast, blurring of the vascular wall, and profound attenuation of the diaphragm. According to the findings of ultrasound examination patient group with NASH was divided into three; as minimal, moderate and marked subgroup parallel to the increase in echogenicity ${ }^{18}$. The subjects were randomly allocated to the experimental group or control group. Each subject was given an envelope containing two cards and was instructed to blindly draw one card on each occasion. This study was single blinded: the person undertaking the assessment and data analysis was unaware of the group of each patient while the subjects and their therapists were not blinded to the treatment group. Patients were included into 2 equal groups; the first group (A) received aerobic exercise training in addition to diet regimen for 3 months, where the second group (B) received no treatment intervention. The study was approved by the Ethics Committee of King Abdulaziz University Hospital, Jeddah, Saudi Arabia and all patients signed informed consent for participation in this study.

\section{Equipment}

1) Treadmill (Track master 400E, gas fitness system, England) was used in performance of walking exercise. 2) Commercial kits (Roche Diagnostics, Geneve, Switzerland) were used to measure serum ALT, AST levels; however commercial kits (Siemens Healthcare Diagnostics, Deerfield, USA) were used to measure IL-6, IL-8 and TNF- $\alpha$ level. Also, kits (Bioclin, Quibasa, Belo Horizonte, MG, Brazil) were used for measurements of Homeostasis Model Assessment-Insulin Resistance (HOMA-IR) index for insulin sensitivity. Also, Commercial kits (Randox, Tokyo, Japan) with K2EDTA were used to measure leptin, adiponectin, total cholesterol, triacylglycerol, high-density lipoprotein cholesterol and low-density lipoprotein cholesterol.

3) Weight and height scale (JENIX DS 102, Dongsang, South Korea) was used to measure weight and height to calculate the body mass index (BMI). Body mass index was calculated by dividing the weight in kilograms by the square of the height in meters $\left(\mathrm{Kg} / \mathrm{m}^{2}\right)$. According to the WHO classification, a BMI of $<18.5 \mathrm{Kg} / \mathrm{m}^{2}$ is under weight, $18.5-24.9 \mathrm{Kg} / \mathrm{m}^{2}$ is normal $25-29.9 \mathrm{Kg} /$ $\mathrm{m}^{2}$ is overweight. A BMI of $>30 \mathrm{Kg} / \mathrm{m}^{2}$ is classified as obese and this group was further divided into moderate obesity (30-34.9 Kg/m²), severe obesity $(35-39.9 \mathrm{Kg} /$ $\left.\mathrm{m}^{2}\right)$ and very severe obesity $\left(\geq 40 \mathrm{Kg} / \mathrm{m}^{2}\right)$. 


\section{Measurements}

\section{Laboratory analysis:}

Venous blood samples were collected in polystyrene tubes after a 12-h fasting, by venipuncture of the antecubital vein while patients rested in a supine position. The blood samples were transported to a laboratory within $1 \mathrm{~h}$ and centrifuged at $+4{ }^{\circ} \mathrm{C}$ to remove serum $(1000=\mathrm{g}$ for $10 \mathrm{~min})$. Serum ALT, AST levels were assayed using a Cobas Integra 800 analyzer (Roche Diagnostics, Geneve, Switzerland). While IL-6 levels were measured using "Immulite 2000" immunassay analyzer (Siemens Healthcare Diagnostics, Deerfield, USA). However, interleukin- 8 (IL-8) and TNF- $\alpha$ levels were analyzed with ELISA kits using ELISA microplate strip washer (ELX 50), and ELISA microplate reader (ELX 808; BioTek Instruments, USA). Homeostasis Model Assessment-Insulin Resistance (HOMA-IR) index for insulin sensitivity was computed following this equation: fasting glycemia $(\mathrm{mmol} / \mathrm{L}) \mathrm{x}$ fasting insulin $(\mathrm{mI}-$ $\mathrm{U} / \mathrm{L}) / 22.5^{19}$ and was measured by the colorimetric enzymatic method using an automatic spectrophotometer and respective kits for analysis (Bioclin, Quibasa, Belo Horizonte, MG, Brazil). Human insulin has been measured with an insulin kit (Roche Diagnostics, Indianapolis, IN, USA) using a cobas immunoassay analyzer (Roche Diagnostics). Also, plasma sample with K2EDTA was collected after centrifugation (2000× $\mathrm{g}$ for 10 min at $4^{\circ} \mathrm{C}$ ) and stored at $-80^{\circ} \mathrm{C}$ to analyze leptin, adiponectin, total cholesterol, triacylglycerol, high-density lipoprotein cholesterol, low-density lipoprotein cholesterol. All analyses were carried out on a Hitachi 7170 Autoanalyser (Tokyo, Japan) or with commercial kits (Randox).

\section{Evaluation of anthropometric parameters}

All measurements were performed at pre-treatment and after three months at the end of the study. The patients were measured while wearing their undergarments and hospital gowns. Height was measured with a digital stadiometer to the nearest $0.1 \mathrm{~cm}$ (JENIX DS 102, Dongsang, South Korea). Body weight was measured on a calibrated balance scale to the nearest $0.1 \mathrm{~kg}$ (HC4211, Cas Korea, South Korea), and BMI was calculated as BMI $=$ Body weight $/(\text { Height })^{2}$.

All measurements of TNF- $\alpha$, Il-6, IL-8, leptin, adiponectin, HOMA-IR, ALT, AST, TC, LDL-c, TG, HDL-c and BMI were taken before the starting of the study and after three months at the end of the study.

\section{Procedures}

Following the previous evaluation, all patients were divided randomly into the following groups:

1. The training group: Fifty nonalcoholic steatohepatitis patients of both sexes received aerobic exercise training program and diet regimen. The aerobic treadmill-based training program (Track master 400E, gas fitness system, England) was at $65 \%$ to $75 \%$ of the maximum heart rate (HRmax) achieved according to a modified Bruce protocol. After an initial, 5-minute warm-up phase performed on the treadmill at a low load, each endurance training session lasted 30 minutes and ended with 5-minute recovery and relaxation phase according to recommendation of American College of Sports Medicine regarding the aerobic exercise application $^{20}$, three sessions / week (i.e. a total of 36 sessions over a 3-month period). Also, the interview-based food survey was performed by dieticians to specify previous food habits and possible anomalies in dietary behavior. The prescribed low calorie diet was balanced, with 15\% as protein, 30 to $35 \%$ as fat and 50 to $55 \%$ as carbohydrate, on average, in order to provide about 1200 Kilocalories/day for three months ${ }^{21}$.

2. The control group: Fifty non-alcoholic steatohepatitis patients of both sexes were asked to maintain their ordinary current life style.

\section{Statistical analysis}

The mean values of TNF- $\alpha$, IL-6, IL-8, leptin, adiponectin, HOMA-IR, ALT, AST, TC, LDL-c, TG, HDL-c and BMI obtained before and after three months in both groups were compared using paired " $t$ " test. Independent " $t$ " test was used for the comparison between the two groups $(\mathrm{P}<0.05)$. Pearson's product moment correlation coefficients (r) were applied to examine the degree of correlation between changes in BMI, ALT and AST levels.

\section{Results}

One hundred NASH patients completed the screening evaluation and underwent randomization. The baseline characteristics of the patients who underwent randomization are shown in Table (1). Most patients (70\%) were men. Fifty patients were assigned to aerobic exercise and diet regimen ( $\mathrm{n}=50 ; 34$ males and 16 females) and control group ( $\mathrm{n}=50,36$ males and 14 females). None of the baseline characteristics differed significantly between the two groups as listed in table (1). 
Table (1): Mean value of demographic data and health state for patients in both groups.

\begin{tabular}{|c|c|c|c|}
\hline & \multicolumn{2}{|l|}{ Mean +SD } & \multirow[t]{2}{*}{ P-value } \\
\hline & NASH & Control & \\
\hline Age (year) & $50.78 \pm 5.34$ & $51.12 \pm 5.86$ & 0.098 \\
\hline Gender ratio (male/female) & $34 / 16$ & $36 / 14$ & 0.271 \\
\hline Weight (kg) & $93.24 \pm 6.51$ & $92.85 \pm 6.22$ & 0.152 \\
\hline Height (cm) & $173.11 \pm 7.82$ & $172.35 \pm 6.95$ & 0.084 \\
\hline BMI $\left(\mathrm{kg} / \mathrm{m}^{2}\right)$ & $32.35 \pm 2.54$ & $31.76 \pm 2.92$ & 0.093 \\
\hline Waist-hip ratio & $0.97 \pm 0.15$ & $0.96 \pm 0.06$ & 0.125 \\
\hline Systolic BP, mm Hg & $141.26 \pm 12.56$ & $140.87 \pm 11.93$ & 0.157 \\
\hline Diastolic BP, mm Hg & $89.31 \pm 8.64$ & $88.75 \pm 10.21$ & 0.162 \\
\hline HbA1C, \% & $5.28 \pm 0.96$ & $5.6 \pm 0.84$ & 0.063 \\
\hline Bilirubin level, mol/L & $13.83 \pm 3.41$ & $14.25 \pm 4.3$ & 0.921 \\
\hline Albumin level, g/L & $43.57 \pm 2.73$ & $42.78 \pm 3.11$ & 0.073 \\
\hline
\end{tabular}

BMI: Body mass index; BP: Blood pressure; HBA1c: Glcocylated hemoglobin

Further, a significant decrease $(\mathrm{P}<0.05)$ in the anthropometric measurements including BMI was observed with the lifestyle intervention group of NASH pa- tients who received dietary and exercise therapy for 3 months, compared to NASH patients of control group (see Table 2).

Table (2): Mean value and significance of BMI, TNF- $\alpha$, IL-6, IL-8, ALT, AST, TC,LDL-c, HDLc, TG, HOMA-IR in group (A) before and after treatment.

\begin{tabular}{|c|c|c|c|}
\hline & \multicolumn{2}{|l|}{ Mean +SD } & \multirow[t]{2}{*}{ P-value } \\
\hline & Before & After & \\
\hline BMI $\left(\mathrm{kg} / \mathrm{m}^{2}\right)$ & $32.35 \pm 2.54$ & $29.15 \pm 2.48^{*}$ & 0.0035 \\
\hline TNF- $\alpha(\mathrm{pg} / \mathrm{mL})$ & $12.76 \pm 2.21$ & $8.21 \pm 2.35^{*}$ & 0.0083 \\
\hline IL-6 (pg/mL) & $5.26 \pm 1.52$ & $3.40 \pm 1.17^{*}$ & 0.0077 \\
\hline IL-8 (pg/mL) & $18.53 \pm 3.78$ & $15.16 \pm 3.35^{*}$ & 0.0056 \\
\hline Leptin $\mathrm{Ng} / \mathrm{ml}$ & $41.64 \pm 5.24$ & $36.68 \pm 4.76^{*}$ & 0.0043 \\
\hline Adiponectin $(\mu \mathrm{g} / \mathrm{mL})$ & $10.51 \pm 3.73$ & $14.96 \pm 3.54^{*}$ & 0.0034 \\
\hline $\operatorname{ALT}(\mathrm{U} / \mathrm{L})$ & $46.32 \pm 4.65$ & $34.65 \pm 4.13^{*}$ & 0.0035 \\
\hline $\operatorname{AST}(\mathrm{U} / \mathrm{L})$ & $44.71 \pm 5.53$ & $35.22 \pm 4.97^{*}$ & 0.0023 \\
\hline TC (mg/dl) & $196.11 \pm 12.42$ & $171.63 \pm 11.04^{*}$ & 0.0019 \\
\hline LDL-c (mg/dl) & $136.52 \pm 10.84$ & $118.31 \pm 11.15^{*}$ & 0.0024 \\
\hline TG (mg/dl) & $157.14 \pm 11.06$ & $124.17 \pm 10.43^{*}$ & 0.0018 \\
\hline HDL-c (mg/dl) & $34.65 \pm 2.87$ & $38.11 \pm 2.32 *$ & 0.0037 \\
\hline HOMA-IR & $4.92 \pm 2.36$ & $2.95 \pm 1.98^{*}$ & 0.0026 \\
\hline
\end{tabular}

BMI: Body mass index; TNF- $\alpha$ : tumor necrosis factor - alpha; IL-6: Interleukin-6; IL-8: Interleukin-8; ALT: Alanine aminotransferase; AST: Aspartate aminotransferase; TC: Total cholesterol; LDL-c: Low density lipoprotein cholesterol; HDL-c: High density lipoprotein cholesterol; TG: Triglyceride; HOMA-IR: Homeostasis Model Assessment-Insulin Resistance Index;* Significant level $(\mathrm{p}<0.05)$.

While at the same time, HDL-C level was somewhat reduced among the control group of NASH patients. On the other hand, the LDL-C level decreased among lifestyle intervention group, whereas an increasing trend was observed for the control group of NASH patients, as LDL-C considered to be bad cholesterol with levels of less than 130 normally recommended. The TC of lifestyle intervention group could be maintained very close to its recommended level of 200 throughout the treatment duration, while TC was found to be slightly increased among the control NASH patients which might indicate a potential risk for atherosclerosis development by accumulation of cells containing excess lipids within the arterial wall that are closely related to NASH development(see Table 2 and 3). 
Table (3): Mean value and significance of BMI, TNF- $\alpha, I L-6$, IL-8, ALT, AST,TC,LDL-c, HDLc, TG, HOMA-IR in group (B) before and after the study.

\begin{tabular}{|c|c|c|c|}
\hline & Mean +SD & & P-value \\
\hline & Before & After & \\
\hline BMI $\left(\mathrm{kg} / \mathrm{m}^{2}\right)$ & $31.76 \pm 2.92$ & $31.98 \pm 2.87$ & 0.126 \\
\hline TNF- $\alpha(\mathrm{pg} / \mathrm{mL})$ & $12.98 \pm 2.37$ & $13.54 \pm 2.56$ & 0.114 \\
\hline IL-6 (pg/mL) & $5.76 \pm 1.71$ & $5.98 \pm 1.95$ & 0.137 \\
\hline IL-8 (pg/mL) & $18.85 \pm 3.26$ & $19.23 \pm 3.57$ & 0.210 \\
\hline Leptin $\mathrm{Ng} / \mathrm{ml}$ & $41.57 \pm 5.13$ & $42.11 \pm 4.92$ & 0.251 \\
\hline Adiponectin $(\mu \mathrm{g} / \mathrm{mL})$ & $10.36 \pm 3.15$ & $10.85 \pm 2.97$ & 0.134 \\
\hline $\operatorname{ALT}(U / L)$ & $46.53 \pm 4.27$ & $46.94 \pm 4.62$ & 0.116 \\
\hline $\operatorname{AST}(\mathrm{U} / \mathrm{L})$ & $44.80 \pm 5.53$ & $45.17 \pm 5.21$ & 0.131 \\
\hline TC (mg/dl) & $195.83 \pm 11.54$ & $196.85 \pm 10.88$ & 0.315 \\
\hline LDL-c (mg/dl) & $137.11 \pm 10.16$ & $137.92 \pm 10.73$ & 0.271 \\
\hline TG (mg/dl) & $158.45 \pm 11.32$ & $159.26 \pm 11.17$ & 0.127 \\
\hline HDL-c (mg/dl) & $35.21 \pm 2.33$ & $34.85 \pm 2.62$ & 0.113 \\
\hline HOMA-IR & $4.48 \pm 2.17$ & $4.75 \pm 2.15$ & 0.254 \\
\hline
\end{tabular}

BMI: Body mass index; TNF- $\alpha$ : tumor necrosis factor - alpha; IL-6: Interleukin-6; IL-8: Interleukin-8; ALT: Alanine aminotransferase; AST: Aspartate aminotransferase; TC: Total cholesterol; LDL-c: Low density lipoprotein cholesterol; HDL-c: High density lipoprotein cholesterol; TG: Triglyceride; HOMA-IR: Homeostasis Model Assessment-Insulin Resistance Index.

Considerable changes were observed in the serum lipid profiles of the NASH patients within the lifestyle intervention group of NASH patients; the results are shown in Table 4. The concentration of TG in the blood that usually provides energy for different metabolic processes was effectively controlled among the lifestyle inter- vention group, whereas the TG level was higher for the control group even after 3 months of dietary and exercise therapy. An increase in HDL-C level was observed in lifestyle intervention group, which is a good indicator of the improvement in NASH patients because its high level seems to be associated with low incidence of coronary heart diseases.

Table (4): Table (2): Mean value and significance of BMI, TNF- $\alpha$, IL-6, IL-8, ALT, AST,TC,LDL-c, HDL-c, TG, HOMA-IR in group (A) and group (B) after the study.

\begin{tabular}{|c|c|c|c|}
\hline & \multicolumn{2}{|l|}{ Mean +SD } & \multirow[t]{2}{*}{ P-value } \\
\hline & Group (A) & Group (B) & \\
\hline BMI $\left(\mathrm{kg} / \mathrm{m}^{2}\right)$ & $29.15 \pm 2.48^{*}$ & $31.98 \pm 2.87$ & 0.0046 \\
\hline TNF- $\alpha(\mathrm{pg} / \mathrm{mL})$ & $8.21 \pm 2.35^{*}$ & $13.54 \pm 2.56$ & 0.0091 \\
\hline IL-6 (pg/mL) & $3.40 \pm 1.17^{*}$ & $5.98 \pm 1.95$ & 0.0083 \\
\hline IL-8 (pg/mL) & $15.16 \pm 3.35^{*}$ & $19.23 \pm 3.57$ & 0.0057 \\
\hline Leptin $\mathrm{Ng} / \mathrm{ml}$ & $36.68 \pm 4.76^{*}$ & $42.11 \pm 4.92$ & 0.0052 \\
\hline Adiponectin $(\mu \mathrm{g} / \mathrm{mL})$ & $14.96 \pm 3.54^{*}$ & $10.85 \pm 2.97$ & 0.0038 \\
\hline $\operatorname{ALT}(\mathrm{U} / \mathrm{L})$ & $34.65 \pm 4.13^{*}$ & $46.94 \pm 4.62$ & 0.0044 \\
\hline $\operatorname{AST}(\mathrm{U} / \mathrm{L})$ & $35.22 \pm 4.97 *$ & $45.17 \pm 5.21$ & 0.0038 \\
\hline TC (mg/dl) & $171.63 \pm 11.04 *$ & $196.85 \pm 10.88$ & 0.0026 \\
\hline LDL-c (mg/dl) & $118.31 \pm 11.15^{*}$ & $137.92 \pm 10.73$ & 0.0029 \\
\hline TG (mg/dl) & $124.17 \pm 10.43^{*}$ & $159.26 \pm 11.17$ & 0.0022 \\
\hline HDL-c (mg/dl) & $38.11 \pm 2.32^{*}$ & $34.85 \pm 2.62$ & 0.0051 \\
\hline HOMA-IR & $2.95 \pm 1.98^{*}$ & $4.75 \pm 2.15$ & 0.0063 \\
\hline
\end{tabular}

BMI: Body mass index; TNF- $\alpha$ : tumor necrosis factor - alpha; IL-6: Interleukin-6;IL-8: Interleukin-8; ALT: Alanine amino transferase; AST: Aspartate amino transferase; TC: Total cholesterol; LDL-c: Low density lipoprotein cholesterol; HDL-c: High density lipoprotein cholesterol; TG: Triglyceride; HOMA-IR: Homeostasis Model Assessment-Insulin Resistance Index;* Significant level (p<0.05). 
In the lifestyle intervention group of NASH patients, the values of leptin, TNF- $\alpha$, IL6, IL8 and HOMA-IR were considerably reduced to significant levels over the period of therapy. While, the levels of these parameters were continuously increased in the control group of NASH patients. Also, as is evident from Tables 2, 3 and
4, the lifestyle intervention group of NASH patients had significant change in levels of AST and ALT from baseline, however ; there was a progressive rise in AST and ALT levels for the control group NASH patients. Moreover, there was a positive relationship between weight changes and levels of AST and ALT in both groups( Tables 5 and 6).

Table (5): The Pearson's correlation coefficients test values and the relationship between the BMI, ALT, AST in group (A).

\begin{tabular}{|l|r|r|}
\hline Test & Pearson's value & $\begin{array}{l}\text { Test Pearson's value Relationship } \\
\text { to body mass index }\end{array}$ \\
\hline Alanine aminotransferase & 0.82 & Strong direct relationship \\
\hline $\begin{array}{l}\text { Aspartate } \\
\text { aminotransferase }\end{array}$ & 0.81 & Strong direct relationship \\
\hline
\end{tabular}

BMI : Body mass index ; ALT :Alanine aminotransferase ; AST : Aspartate aminotransferase

Table (6): The Pearson's correlation coefficients test values and the relationship between theBMI, ALT, AST in group (B).

\begin{tabular}{|l|r|r|}
\hline Test & Pearson's value & $\begin{array}{l}\text { Test Pearson's value Relationship } \\
\text { to body mass index }\end{array}$ \\
\hline Alanine aminotransferase & 0.83 & Strong direct relationship \\
\hline $\begin{array}{l}\text { Aspartate } \\
\text { aminotransferase }\end{array}$ & 0.80 & Strong direct relationship \\
\hline
\end{tabular}

BMI : Body mass index ; ALT :Alanineaminotransferase ; AST : Aspartate aminotransferase

\section{Discussion}

The aim of this study was to detect changes in biochemical parameters following weight loss in patients with non-alcoholic steatohepatitis. The mean values of leptin, TNF- $\alpha$, IL6, IL8, ALT, AST, HOMA-IR, TC, LDL-c, TG and BMI were significantly decreased in group (A), where the mean value of adiponectin and HDL-c were significantly increased, while there were no significant changes in group (B). Also; there was a significant difference between both groups at the end of the study, these findings are supported and agreed by several previous studies.

There is a growing concern for NASH as the next big therapeutic challenge in hepatology because of the possible evolution of NASH toward end-stage liver disease, as well as the need for liver transplantation. The novel of this study is that although exercise and diet improvement may reduce the overall magnanimity of in- sulin resistance, hyperlipidemia and abnormal cytokine metabolism, there has been only limited research on the effects of weight reduction as the sole intervention on these abnormal biochemical parameters in individuals with NASH. However, the limitation of this study is no recording of the histological changes to the treatment intervention

Results of our study was confirmed with Dandona et al. who reported that weight loss reduces TNF- $\alpha$ in the obese ${ }^{22}$. Also, Sandoval and Davis approved that patients who had bariatric surgery gained reduction in IL-6 concentration and improved insulin sensitivity in parallel to weight $\operatorname{loss}^{23}$. However, Chu and colleagues confirmed that serum leptin level was reduced after weight reduction program ${ }^{24}$. Also, Arita and co-authors found that exercise training resulted in both reduction in serum leptin and improved leptin sensitivity ${ }^{25}$. Ruche and $\mathrm{McD}$ onald confirmed that weight loss induces adiponectin synthesis ${ }^{26}$. 
Ueno and colleagues conducted their study of weight reducing program on thirty-five obese NIDDM patients for twelve weeks (diet restriction and exercise) and confirmed that improved serum leptin, lipoprotein profile, insulin sensitivity and glucose control were associated with weight $\operatorname{loss}^{27}$. Tilg and Kaser in their previous study on twenty-five obese patients with fatty liver on three months of restricted diet and exercise, concluded that weight reduction was associated with reduction in serum levels of aminotransferase, albumin, total cholesterol and fasting blood glucose $\mathrm{e}^{28}$.

\section{Conclusion}

Weight loss modulates insulin resistance, adiponectin, leptin, inflammatory cytokines and markers of hepatic function in patients with non-alcoholic steatohepatitis.

\section{Acknowledgment}

This project was funded by the Deanship of Scientific Research (DSR), King Abdulaziz University, Jeddah, under grant no.(324/290/1434). The authors, therefore, acknowledge with thanks DSR technical and financial support.

\section{References}

1. Ji G, Yang Q, Hao J, Guo L, Chen X, Hu J, Leng L, Jiang Z. Anti-inflammatory effect of genistein on non-alcoholic steatohepatitis rats induced by high fat diet and its potential mechanisms. International Immunopharmacology.2011; 11(6): 762-768.

2. Bellentani S, Marino M. Epidemiology and natural history of non-alcoholic fatty liver disease (NAFLD). Ann. Hepatol. 2009; 8: S4-S8.

3. Amarapurkar D, Hashimoto E, Lesmana L, Sollano J, Chen P, Goh K. How common is non-alcoholic fatty liver disease in the Asia-Pacific region, and are there local differences?. J. Gastroenterol Hepatol. 2007; 22: 788 793.

4. Harrison S, Kadakia S, Lang K, Schenker S. Nonalcoholic steatohepatitis: what we know in the new millennium. Am J Gastroenterol. 2002; 97: 2714-2724.

5. Musso G, Gambino R, Cassader M, Pagano G. A meta-analysis of randomized trials for the treatment of nonalcoholic fatty liver disease. Hepatology. 2010; 52: 79_ 104.

6. Duvnjak M, Lerotić I, Baršić N, Tomašić V, Jukić L, Velagić V. Pathogenesis and management issues for non-alcoholic fatty liver disease. World J Gastroenterol. 2007; 13: 4539-4550.

7. Agarwal N, Sharma B. Insulin resistance and clinical aspects of non-alcoholic steatohepatitis (NASH). Hepatology Research. 2005; 33(2): 92-96.

8. Tarantino G, Saldalamacchia G, Conca P, Arena A. Non-alcoholic fatty liver disease: further expression of the metabolic syndrome. J Gastroenterol Hepatol. 2007; 22: 293-303.

9. Uysal S, Armutcu F, Aydogan T, Akin K, Ikizek M, Yigitoglu M. Some inflammatory cytokine levels, iron metabolism and oxidan stress markers in subjects with nonalcoholic steatohepatitis. Clinical Biochemistry. 2011; 44(17-18): 1375-1379.

10. Koek G, Liedorp P, Bast A. The role of oxidative stress in non-alcoholicsteatohepatitis. Clin Chim Acta. 2011; 412: 1297-1305.

11. Ratziu V, Bellentani S, Cortez-Pinto H, Day C, Marchesini G. A position statement on NAFLD/ NASH based on the EASL 2009 special conference. $J$ Hepatol. 2009; 53: 372-384.

12. Hebbard L, George J. Animal models of nonalcoholic fatty liver disease. Nat Rev Gastroenterol Hepatol. 2011; 8: 34-44.

13. Garg A, Misra A. Hepatic steatosis, insulin resistance, and adipose tissue disorders. J Clin Endocrinol Metab. 2002; 87: 3019-3022.

14. Diehl A. Nonalcoholic steatohepatitis. Semin Liver Dis. 1999; 19: 221-229.

15. Schindhelm R, Diamant M, Dekker J, Tushuizen M, Teerlink T, Heine R. Alanine aminotransferase as a marker of non-alcoholic fatty liver disease in relation to type 2 diabetes mellitus and cardiovascular disease. Diabetes Metab Res Rev. 2006; 22: 437-443

16. Miele L, Forgione A, Hernandez A, Gabrieli M, Vero V, Di Rocco P. The natural history and risk factors for progression of non-alcoholic fatty liver disease and steatohepatitis. Eur Rev Med Pharmacol Sci. 2005; 9: 273-278.

17. Paradis V, Perlemuter G, Bonvoust F, et al. High glucose and hyperinsulinemia stimulate connective tissue growth factor expression: a potential mechanism involved in progression to fibrosis in nonalcoholic steatohepatitis. Hepatology.2001;34(4 Pt 1):738-44.

18. Saadeh S, Younossi Z, Remer E, Gramlich T, Ong J, Hurley M. The utility of radiological imaging in non $\neg$ alcoholic fatty liver disease. Gastroenterology. 2002; 123(3): 745-750.

19. Wilund KR. Is the anti-inflammatory effect of regular exercise responsible for reduced cardiovascular disease? Clin Sci (Lond). 2007; 112(11):543-55.

20. American College of Sports Medicine .Guidelines for graded exercise testing and exercise prescription, Lea \& Febiger, Philadelphia. 2005. 
21. World Health Organization: Diet, Nutrition and the Prevention of Chronic Diseases. London, HMSO. 1990 (Tech. Rep. Ser., no. 797)

22. Dandona P, Weinstock R, Thusu K, Abdel-Rahman E, Aljada A, Wadden T. Tumor necrosis factor- $\alpha$ in serum of obese patients: fall with weight loss. J Clin Endocrinol Metab. 1998 Aug;83(8):2907-10.

23. Sandoval D, Davis S. Leptin, Metabolic control and regulation. J. Diabetes Complications. 2003; 17(2): 108-13. 24. Chu N, Stampfer M, Splegelman D. Dietary and lifestyle factors in relation to plasma leptin concentrations among normal weight and overweight men. Int J Obes Relat Metab Dis. 2001; 25: 106-114.
25. Maeda N, Takahashi M, Funahashi T, et al. PPARgamma ligands increase expression and plasma concentrations of adiponectin, an adipose-derived protein. Diabetes.2001 ;50(9):2094-9.

26. Ruche R, McDonald R. Use of antioxidants nutrients in the prevention and treatment of type 2 diabetes. J Coll Nutr. 2001; 20: S 363 - S 369.

27. Ueno T, Sugawara H, Sujaku K, et al. (1997): Therapeutic effects of restricted diet and exercise in obese patients with fatty liver. J Hepatol. 1997;27(1):103-7.

28. Tilg H, Kaser A. Treatment strategies in nonalcoholic fatty liver disease. Nat Clin Pract Gastroenterol Hepatol. 2005; 2:148-155. 